\title{
Different Modes of Synergistic Toxicities between Metam/Copper (II) and Metam/Zinc (II) in HepG2 Cells: Apoptosis vs. Necrosis
}

\author{
Rui-mei Fan, ${ }^{1,2}$ Ben-zhan Zhu, ${ }^{1}$ Chin-pao Huang, ${ }^{2}$ Zhi-guo Sheng, ${ }^{1}$ Li Mao, ${ }^{1}$ Ming-xin Li ${ }^{2}$ \\ ${ }^{1}$ State Key Laboratory of Environmental Chemistry and Ecotoxicology, Research Center for \\ Eco-Environmental Sciences, Chinese Academy of Sciences, Beijing 100085, China \\ ${ }^{2}$ Environmental Engineering, Civil and Environmental Engineering Department, \\ University of Delaware, USA
}

Received 31 July 2015; revised 11 September 2015; accepted 13 September 2015

\begin{abstract}
Both metam sodium and copper/zinc-containing compounds are widely used as fungicides. They therefore may co-occur in the biosphere. Despite certain studies of individual toxicity for either metam or copper (II)/zinc (II), their synergistic toxicity has not been examined. In this paper, a remarkable synergistic toxicity was observed in HepG2 cells when metam and copper (II)/zinc (II) at non-toxic and sub-toxic levels were combined. Unexpectedly, cell death modes between metam/copper (II) and metam/zinc (II) were different: For metam/copper (II), apoptosis was evident from morphological characteristics including cytoplasm-chromatin condensation, phosphatidylserine (PS) exposure, SubG ${ }_{0} / G_{1} D N A$ fragmentation, mitochondrial membrane potential decrease, pro/anti-apoptotic protein activation, and cytochrome c release; for metam/zinc (II), necrosis was evident from organelle swelling and uncontrolled collapse. To our knowledge, this work first not only demonstrates the synergistic toxicities of metam and both copper (II)/zinc (II), but also verifies the different modes of apoptosis/necrosis between metam/copper (II) and metam/zinc (II). (c) 2015 Wiley Periodicals, Inc. Environ Toxicol 31: 1964-1973, 2016.
\end{abstract}

Keywords: metam; copper (II); zinc (II); synergistic toxicity; apoptosis; necrosis

\section{INTRODUCTION}

Since metam sodium was first registered in 1973, it has been widely used as an herbicide, pesticide, fungicide, and soil fumigant. Because it is the simplest member of the dithioca-

Additional Supporting Information may be found in the online version of this article.

Correspondence to: Ben-zhan Zhu, Professor in State Key Laboratory of Environmental Chemistry and Ecotoxicology, Research Center for EcoEnvironmental Sciences, Chinese Academy of Sciences, Beijing 100085, China.Email: bzhu@rcees.ac.cn; Tel: 01062849030.

Contract grant sponsor: National Natural Science Foundation of China, State Key Laboratory of Environmental Chemistry and Ecotoxicology, Research Center for Eco-Environmental Sciences, and Chinese Academy of Sciences.

Published online 29 September 2015 in Wiley Online Library (wileyonlinelibrary.com). DOI: 10.1002/tox.22197 bamates, its structure is unstable. In soil, metam can be rapidly decomposed into methyl isothiocyanate (MITC), which can react with amines and thiols in biological molecules when applied as a soil fumigant, a pre-emergent herbicide, fungicide, or insecticide.

As an alternative to methyl bromide for weed control, the toxicity of metam is relatively low. It ranks third on the EPA's list of the most commonly used conventional pesticides (by weight) in the U.S. agricultural crop production, with $50 \sim 55$ million pounds used in 2007 (USEPA, 2006-2007). In China, its application is expanding, making it a domestic pesticide along with worldwide agricultural modernization.

However, due to an accident that caused approximately 19 000 gallons of metam sodium to be spilled into the Sacramento River, more attention was focused on its negative effects on the environment and human health. Recent reports indicate that metam sodium can be converted into MITC in 
the human stomach. Yet, only $80 \%$ of the MITC was detected in the urine, which suggests that the remaining metam can be absorbed intact in the colon (California Environmental Protection Agency Sacramento). Also, metam sodium was found to cause the dysfunction of neuronal and muscular development of zebrafish (Haendel et al., 2004; Tilton et al., 2006; Tilton and Tanguay, 2008). Pruett et al. reported that metam can lead to several dysfunctions of the immune system in mice, such as thymic atrophy and reduced natural killer cell activity (Padgett and Pruett, 1992; Pruett et al. 1992; Keil et al.1996). Additionally, environmental and occupational exposure to metam by humans can result in numerous symptoms such as rash, headache, nausea, salivation, dizziness, and asthma (Lee et al., 2002). Notably, the incidence rate of leukemia among California-born children was highly associated with probable and possible carcinogen-metam use (Reynolds et al., 2005).

In general, metam is either sprayed on the surface of vegetables or injected into the soil. Likewise, numerous copper/ zinc containing fungicides, such as ziram and zineb, are found in agricultural lands. Especially, as food additives and wood preservatives, copper/zinc containing compounds also appear in water near farmland and at timber disposal areas as well as in bodily fluids and tissues from ordinary people. Therefore, they can co-exist in the environment.

However, current studies mainly are conducted on the individual toxicity of either metam sodium or copper (II)/ zinc (II). To our knowledge, few studies for their combined toxicity have been published. Because of its unique function and anatomical location, the liver, frequently exposed to a multitude of toxicants for detoxification, is highly susceptible to tissue injury. To this end, we explored the synergistic toxicity in HepG2 cell as a typical model.

An important process of cytotoxicity follows the pathway of programmed cell death. The conventional view refers to programmed cell death via caspase-mediated apoptosis. In contrast to necrosis, it is considered as an accidental and unwanted cell demise, which is carried out in a non-regulated manner and caused by extreme condi-

\section{ABBREVIATIONS}

$\begin{array}{ll}\text { DAPI } & \text { 4 }^{\prime} \text {,6-diamidino-2-phenylindole } \\ \text { DMDC } & \text { dimethyl dithiocarbamate } \\ \text { DSF } & \text { disulfiram } \\ \text { FITC } & \text { annexin V-FITC } \\ \text { GSH } & \text { reduced glutathione } \\ \text { GSSG } & \text { oxidized glutathione } \\ \text { metam } & \text { metam sodium } \\ \text { MTT } & \text { 3-(4,5-dimethylthiazol-2-yl)-2,5-diphenyltetrazo- } \\ & \text { lium bromide } \\ \text { PDTC } & \text { ammonium pyrrolidine dithiocarbamate } \\ \text { PBS } & \text { phosphate buffered saline } \\ \text { PI } & \text { propidium iodide }\end{array}$

tions. However, few researchers differentiate between specific drugs in certain cells. Therefore, we combined an integrated analysis of various factors addressing molecular and cellular knowledge to provide a better understanding of toxicants as the stimuli that initiate intracellular events, ultimately leading to apoptosis or necrosis. Recently, some pathologists advanced the term necroptosis, which is also described as a possible programmed process with morphological resemblance to necrosis (Nikoletopoulou, 2013; Linkermann and Green, 2014). Therefore, necroptosis has been defined as a programmed form of necrosis (Pasparakis and Vandenabeele, 2015). Although necroptosis has been raised as an emerging type of cell death in liver diseases, there has been little research on the involvement of necroptosis rather than necrosis (Guicciardi et al., 2013; Saeed and Jun, 2014). In this paper, apoptosis and necrosis are discussed with HepG2 cell lines induced by metam/copper (II) or metam/ zinc (II).

\section{EXPERIMENTAL PROCEDURES}

\section{Chemicals}

All chemical regents were purchased from Sigma-Aldrich ${ }^{\circledR}$, except those otherwise noted. MTT was purchased from Amesco ${ }^{\circledR}$. Cell culture reagents were purchased from GibcoBRL $^{\circledR}$ Life Technologies. Annexin V-FITC Apoptosis Detection Kit was purchased from BD Pharmingen. F-actin phalloidin staining was purchased from Alexis ${ }^{\circledR}$ Corporation. Antibodies against caspase-3, caspase-9, cytochrome C, Bcl2, and Bax were purchased from $\mathrm{CST}^{\circledR}$. Horseradish peroxidase-conjugated secondary antibodies were obtained from Santa Cruz ${ }^{\circledR}$ Biotechnology. Western blot enhanced chemiluminescence (ECL) reagent was purchased from Amersham $^{\circledR}$.

\section{Cell Growth Culture and Treatment}

HepG2 cells were cultured in RPMI-1640 containing 10\% fetal bovine serum (FBS), supplemented with $100 \mathrm{U} / \mathrm{mL}$ penicillin and $100 \mu \mathrm{g} / \mathrm{mL}$ streptomycin, and $2 \mathrm{mM}$ l-glutamine in a humidified atmosphere with $5 \% \mathrm{CO}_{2}$, at $37^{\circ} \mathrm{C}$. About $100 \mathrm{mM}$ metam sodium, copper (II) sulfate, and zinc (II) sulfate stock were diluted with cell growth culture to an effective concentration for HepG2 cells.

\section{Cell Viability Assays}

Cell viability was measured by the MTT assay. One million cells in $200 \mu \mathrm{L}$ of media were seeded in each well of a 96well tissue culture microtiter plate. After incubating for $24 \mathrm{~h}$, the HepG2 cells were exposed to the chemicals overnight. Then the cells were washed with PBS. Subsequently, 100 $\mu \mathrm{L}$ of $0.05 \mathrm{mg} / \mathrm{mL}$ MTT were incubated for $4 \mathrm{~h}$, then 
removed, $100 \mu \mathrm{L}$ dimethyl sulfoxide was extracted from the formed formazan salt and measured at $570 \mathrm{~nm}$ (Hansen et al., 1989).

\section{Phase Contract Microscopy}

HepG2 Cells growing on the slides were fixed by $4 \%$ formaldehyde for $10 \mathrm{~min}$ and then washed twice in PBS. Permeabilization of HepG2 cells membranes was performed in $0.1 \%$ Triton X-100 in PBS for 10 min and then washed twice in PBS. FITC-conjugated phalloidin was used to label Factin for $30 \mathrm{~min}$ in the dark and then cell nuclei were counterstained with DAPI (Liu et al., 2009).

\section{Confocal Microscopy Assays}

HepG2 Cells were grown on the slides, fixed by $4 \%$ formaldehyde for $10 \mathrm{~min}$, and then washed twice in PBS. Permeabilization of cell membrane of HepG2 cells was performed in $0.1 \%$ Triton X-100 in PBS for 10 min and then washed twice in PBS. FITC-conjugated phalloidin staining was used to label F-actin for $30 \mathrm{~min}$ in the dark and then cell nuclei were counterstained with DAPI.

\section{Flow Cytometry Assays}

$5 \mathrm{w}$ ? $>$ HepG2 cells were harvested, washed gently twice in cold PBS, and then suspended in $1 \times$ binding buffer at a concentration of $1 \times 10^{5}$ cells $/ \mathrm{mL}$. Next, the cells were stained with FITC and PI dyes, and were incubated for 15 min before analysis with a FACScan. The $5 \times 10^{5}$ cells were harvested and washed with PBS. The cells were re-suspended in $80 \%$ ethanol and stored at $4^{\circ} \mathrm{C}$ for $24 \mathrm{~h}$, re-washed with PBS, and the pellet was re-suspended in Hank's Balanced Salt Solution (HBSS) containing $50 \mu \mathrm{g} /$ $\mathrm{mL}$ RNase A, $0.1 \mathrm{mM}$ EDTA(Na) $)_{2}$ and $0.1 \%$ Triton X-100 at $37^{\circ} \mathrm{C}, 30 \mathrm{~min}$. The pellets were stained with PI $(50 \mu \mathrm{g} /$ $\mathrm{mL}$ ) for DNA cycle analysis.

\section{Western Blot Assays}

Cells were washed twice with PBS, lysed, and centrifuged. Aliquots were measured for the protein concentration. About $25 \mu \mathrm{g}$ protein extract was loaded onto $15 \%$ sodium dodecyl sulfate polyacrylamide gel electrophoresis (SDS-PAGE). Next, Proteins were transferred to nitrocellulose membrane, which was blocked with 5\% nonfat dry milk for $1 \mathrm{~h}$. Subsequently, primary antibodies Bcl-2, Bax, cleaved caspase 3, and pro caspase- 9 were probed with the membrane at $4^{\circ} \mathrm{C}$ overnight. The membranes were conjugated with secondary antibodies horseradish peroxidase and detected with an enhanced chemiluminescence reagent (Duan et al., 2003).

\section{Real Time RCR Assays}

The isolation of total RNA was performed using a TRIzol kit (Invitrogen) and quantified by the nano-drop machine.

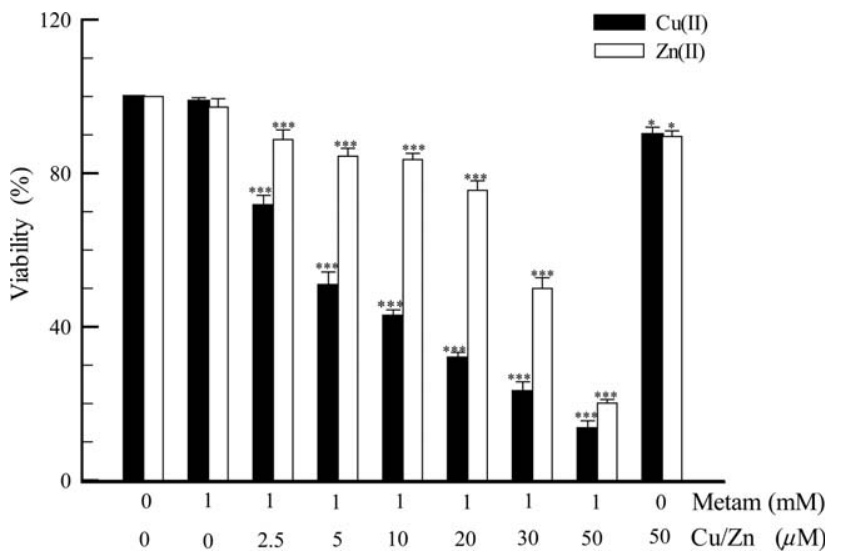

Fig. 1. Synergistic cytotoxicity in HepG2 cells between metam and copper (II)/zinc (II) for $12 \mathrm{~h}$. The dose-dependent concentration of copper (II)/zinc (II) was $0 \sim 50 \mu \mathrm{M}$ when metam fixed at $1 \mathrm{mM}$. Data were presented in independent triplicate experiments. The statistical analysis was followed Turkey-Kramer comparisons at significance level of ${ }^{\star} P<0.05$, ${ }^{\star \star} P<0.01$, or ${ }^{\star * \star} P<0.001$ with respect to the control.

SuperScript II was used to transcribe $1 \mu \mathrm{L}$ of RNA according to the instructions of the manufacturer. The primers of caspase 3, 7, 8, 9, Bcl-2, Bax, DNA fragmentation factor, $40 \mathrm{kDa}$ (DFFB), and DNA fragmentation factor, $45 \mathrm{kDa}$ (DFFA) were designed by software primer 3.0. The primers were amplified by FastStart Universal SYBR Green Master (ROX). The real time-polymerase chain reaction (RT-PCR) results were analyzed on the Startgene MX3005PTM machine. Ct values were normalized to the expression of the housekeeping GAPDH gene.

\section{Statistical Analysis}

The data were expressed as mean \pm SD statistical significance of the difference among treatments. The JMP Pro analytical method was used by one-way analysis of variance and covariance (ANOVA) followed Tukey-Kramer comparisons at significance level of $* P<0.05, * * P<0.01$, or $* * * P<0.001$ with respect to the control.

\section{RESULTS}

\section{Synergistic Toxicity Between Metam and Copper (II)/Zinc (II)}

MTT assays were used to detect the individual cytotoxicity of metam, copper (II)/zinc (II), and the synergistic cytotoxicity between metam and copper (II)/zinc (II) in HepG2 cells. Unexpectedly, a remarkable synergistic toxicity was found between metam sodium and copper (II)/ zinc (II), when either of them alone was non/sub-toxic. Respectively, the $\mathrm{IC}_{50}$ value of metam sodium, copper (II)/zinc (II) alone was $5 \mathrm{mM}, 400 \mu \mathrm{M}$, and $300 \mu \mathrm{M}$. By contrast, in the presence of $1 \mathrm{mM}$ metam sodium, the 
TABLE I. Synergistic toxicity between other dithiocarbamates and metals in HepG2 cells for $12 \mathrm{~h}$. The concentrations of dithiocarbamates; including metam, PDTC, DMDC, and DSF; and the seven metals were 0.1 mM. Each exposure was replicated six times. The data represent the viability of HepG2 cells under the corresponding concentration or combined concentration

\begin{tabular}{lcccc}
\hline & $\begin{array}{c}\text { Metam } \\
\text { Metal ions }\end{array}$ & $\begin{array}{c}\text { PDTC } \\
(97.0 \pm 1.8)\end{array}$ & $\begin{array}{c}\text { DMDC } \\
(74.0 \pm 1.5)\end{array}$ & $\begin{array}{c}\text { DSF } \\
(37.2 \pm 0.9)\end{array}$ \\
\hline $\mathrm{Cu}^{2+}(79.9 \pm 1.2)$ & $2.0 \pm 1.0$ & $19.8 \pm 0.2$ & $15.1 \pm 2.5$ & $21.0 \pm 1.8$ \\
$\mathrm{Zn}^{2+}(99.0 \pm 0.8)$ & $11.4 \pm 0.7$ & $19.8 \pm 0.2$ & $17.6 \pm 0.5$ & $26.9 \pm 2.0$ \\
$\mathrm{Cd}^{2+}(23.2 \pm 1.6)$ & $10.3 \pm 2.1$ & $16.5 \pm 0.1$ & $9.92 \pm 1.1$ & $24.3 \pm 1.0$ \\
$\mathrm{Cr}^{3+}(99.9 \pm 1.3)$ & $73.7 \pm 2.4$ & $94.2 \pm 2.4$ & $88.6 \pm 1.8$ & $43.8 \pm 3.4$ \\
$\mathrm{Ni}^{2+}(77.7 \pm 0.6)$ & $45.5 \pm 1.7$ & $84.6 \pm 3.4$ & $83.1 \pm 3.0$ & $44.1 \pm 3.6$ \\
$\mathrm{Mn}^{2+}(93.8 \pm 3.5)$ & $47.5 \pm 1.1$ & $74.3 \pm 1.8$ & $85.4 \pm 1.5$ & $44.5 \pm 3.5$ \\
$\mathrm{~V}^{2+}(92.2 \pm 2.1)$ & $57.2 \pm 4.0$ & $77.1 \pm 1.0$ & $72.9 \pm 3.7$ & $54.4 \pm 1.3$ \\
\hline
\end{tabular}

$\mathrm{IC}_{50}$ value of copper (II)/zinc (II) in turn were reduced to $5 \mu \mathrm{M}$ and $30 \mu \mathrm{M}$ (as shown in Fig. 1). The $\mathrm{IC}_{50}$ value of metam was decreased to $15 \mu \mathrm{M}$ and $350 \mu \mathrm{M}$, individually, when copper (II)/zinc (II) was fixed at $50 \mu \mathrm{M}$. Moreover, similar synergistic toxicity was observed when $1 \mathrm{mM}$ PDTC was combined with zinc (II) at various concentrations. Also, copper (II)/zinc (II) markedly potentiated cytotoxicity induced by other dithiocarbamates such as PDTC, DMDC, and DSF (as shown in Table I). By contrast, the synergistic toxicity between metam and copper (II) was most remarkable when compared to the other dithoicarbamates, either PSTC, DMDC or DSF, and copper (II) at the concentration of $100 \mu \mathrm{M}$. By comparison, the synergistic toxicity of both metam and copper (II)/ zinc (II) were more significant than that of metam and the other metals such as chromium (III), nickel (II), manganese (II), and vanadium (II). Furthermore, we found that similar synergistic toxicity between metam sodium and copper (II)/zinc (II) ions existed in other cell lines such as human lung cancer cells (A549 cells), mouse monocyte macrophages (Raw264.7 cells), human renal proximal tubule epithelial cells (HK-2 cells), and normal human liver cells (HL-7702 and QSG-7702 cells). The results indicate there is a general spectrum of environmental and human health effects.

\section{Identification of Apoptotic/Necrotic Death Mode Between Metam/Copper (II) and Metam/Zinc (II) in HepG2 Cells}

As shown in Figure 1 in the synergistic toxicity between metam and copper (II)/zinc (II), there was, in HepG2 cells of metam/zinc (II), a sharp conversion from cell survival to death, implying that HepG2 cells probably underwent a necrotic process. In contrast, previous studies regarding dithiocarbamates mainly focused on cell apoptosis through inhibiting enzymatic activity of glutathione reductase (Nobel, et al., 1995; Kim et al., 1999; Daniel et al., 2005;
Milacic et al., 2008). Did HepG2 cells induced by metam/ zinc (II) follow a necrotic death?

\section{Different Morphological Changes in HepG2 Cells Between Metam/Copper (II) and Metam/Zinc (II) by Microscopy}

Two different morphologies were individually observed in metam/copper (II)-treated HepG2 cells and metam/zinc (II)treated HepG2 cells. As shown in Figure S1, Metam/copper (II)-treated cells stained by Wright-Giemsa dyes under a phase-contrast inverted microscope showed typical apoptotic morphologies [as shown in Figs. S1(B,E)], such as rounding up of cells, blebbing of cells, formation of apoptotic bodies, shrinking of cytoplasm, and condensation of chromatin; by contrast, metam/zinc (II)-treated cells displayed classical necrotic morphologies [as shown in Figs. S1(C,F)], such as the appearance of balloon-like structures, rupture of the cytoplasmic membrane, blurring of cell membrane boundaries, and swelling of cytoplasmic organelles. These distinct morphological features of apoptotic versus necrotic features were seen, respectively, in metam/copper (II)-treated cells and metam/zinc (II)-treated cells.

In addition, the most abundant microstructure of the cytoplasm cytoskeleton, cytosolic F-actin, has been demonstrated to be damaged during apoptosis induced by different stimuli (Kroupová et al., 2007). As previously described in Figure S1, metam/copper (II)-treated HepG2 cells underwent morphological alterations typified by a change from their characteristic irregular shape to a rounded shape. Also, widespread blebs were detached from the cell membrane surface. These changes suggested damage to the cytoskeleton; F-actin, monitored using fluorescence of FITC-conjugated phalloidin, displayed reorganization, and disassembly of actin filaments. As shown in Figure 2(A-D), HepG2 cells with control, $1 \mathrm{mM}$ metam, and $0.05 \mathrm{mM}$ copper (II)/zinc (II), had abundant and well-organized stress fibers, compared to either metam/copper (II) or metam/zinc (II)-treated HepG2 cells. On the contrary, noticeable disorganization and 


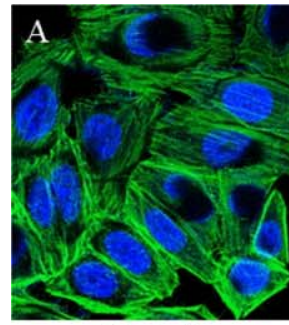

Control

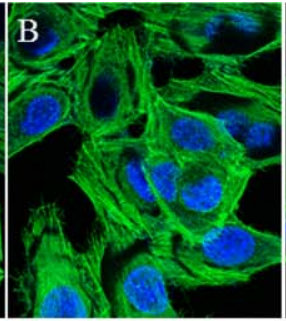

Metam 1mM

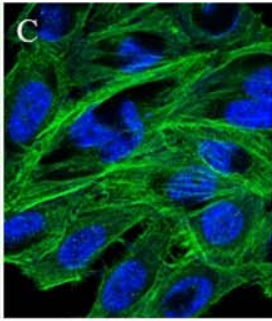

$\mathrm{Cu} 0.05 \mathrm{mM}$

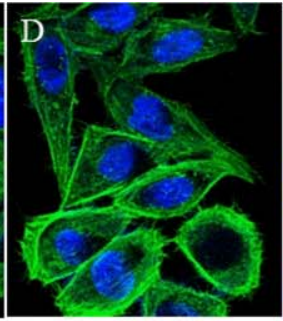

$\mathrm{Zn} 0.05 \mathrm{mM}$

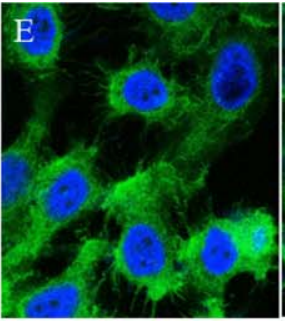

$\mathrm{M} / \mathrm{Cu}$ $(1+0.05) \mathrm{mM}$

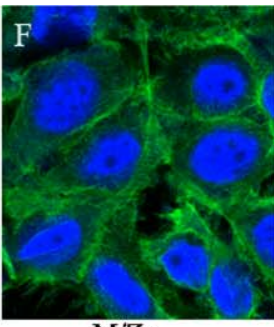

$\mathrm{M} / \mathrm{Zn}$

$(1+0.05) \mathrm{mM}$

Fig. 2. F-actin filaments of apoptosis/necrosis-necroptosis in HepG2 cells treated with metam or/and copper (II)/zinc (II) for $1 \mathrm{~h}$ visualized by $10 \times 40$ Confocal Microscope. M means metam; M/Cu means the mixture of metam/copper (II); and M/Zn means the mixture of metam/zinc (II). [Color figure can be viewed at wileyonlinelibrary.com]

depolymerization of F-actin filaments happened in the HepG2 cells treated with either metam/copper (II) or metam/zinc (II). Further, the morphology of the cytoskeleton in metam-copper (II) treated HepG2 cells was also different from that in metam-zinc (II) treated HepG2 cells. Specifically, fluorescent images of F-actin in metam/copper (II)treated HepG2 cells display short, thick, and punctate clusters concentrated around interphase nuclei, as shown in Figure 2(E), indicating classic apoptotic incidents. Yet, we observed that the arrangement of F-actin cytoskeleton in metam/zinc (II)-treated cells [as shown in Fig. 2(F)], was even thinner and looser than that of control cells, possibly involving a rapid progressively depolymerized necrotic lysis.

\section{Different Phenomena of PS Exposure and DNA Fragmentation in HepG2 Cells Between Metam/Copper (II) and Metam/Zinc (II) by Flow Fluorocytometry}

Phosphatidylserine (PS), as an early molecular marker of apoptosis in the inner leaflet of the plasma membrane, conjugated with annexin V-FITC/PI, can help discriminate apoptosis and necrosis. A possible mechanism for PS exposure could be inactivation of the amino phospholipid translocase, leading to a coordinate increase in phospholipid flip-flop and loss of phospholipid asymmetry (Krysko et al., 2006). Our results show that metam/copper (II)-treated HepG2 cells were divided into an early apoptotic population (lower-right quadrant, PI-negative/annexin V-positive cells) and a late
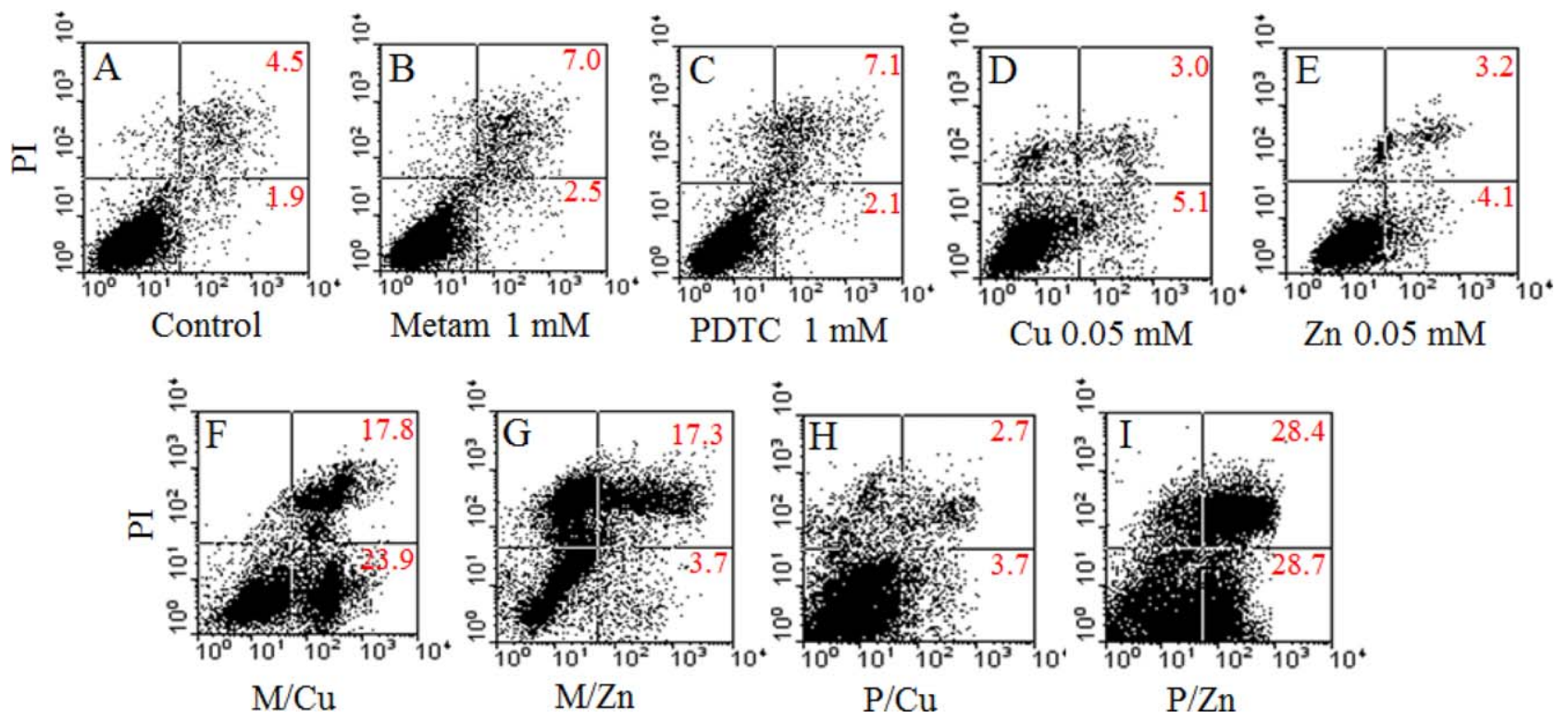

Fig. 3. PS exposure of apoptosis/necrosis-necroptosis in HepG2 cells treated with PDTC/metam or/and copper (II)/zinc (II) for $3 \mathrm{~h}$ analyzed by flow cytometry. Each data for three tubes combined. M means metam; P means PDTC; M/Cu means the mixture of metam/copper (II); M/Zn means the mixture of metam/zinc (II); P/Cu means the mixture of PDTC/copper (II); and $\mathrm{P} / \mathrm{Zn}$ means the mixture of PDTC/zinc (II). [Color figure can be viewed at wileyonlinelibrary.com] 

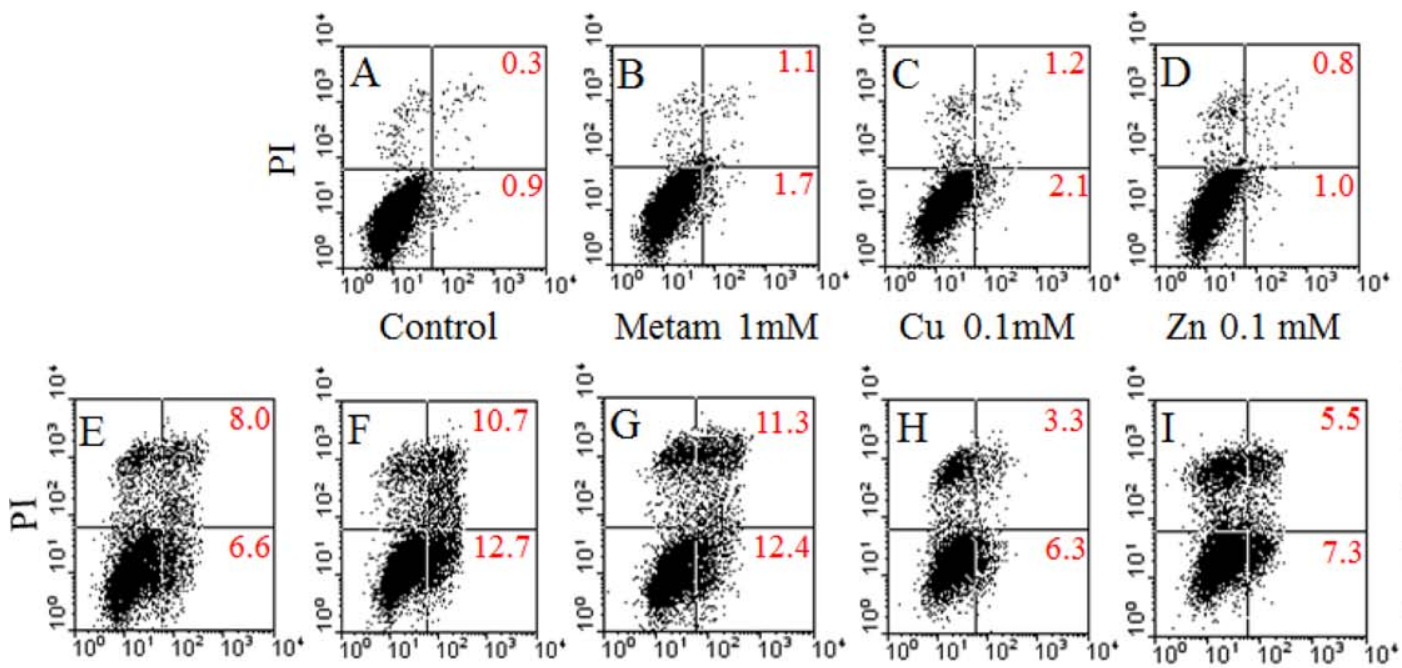

Zn $0.1 \mathrm{mM}$

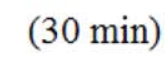

$(60 \mathrm{~min})$

(90 $\mathrm{min})$

(30min)
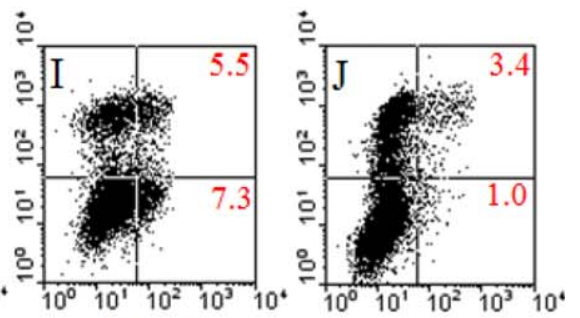

$\mathrm{M} / \mathrm{Cu}$

(60 $\mathrm{min})$

( $90 \mathrm{~min})$

$\mathrm{M} / \mathrm{Zn}$

Fig. 4. Time-course effects on apoptosis/necrosis-necroptosis in HepG2 cells treated with $1 \mathrm{mM}$ metam and $0.1 \mathrm{mM}$ copper (II)/zinc (II) by flow cytometry. Each data for three tubes combined. M means metam; M/Cu means the mixture of metam/copper (II); and M/Zn means the mixture of metam/zinc (II). [Color figure can be viewed at wileyonlinelibrary.com]
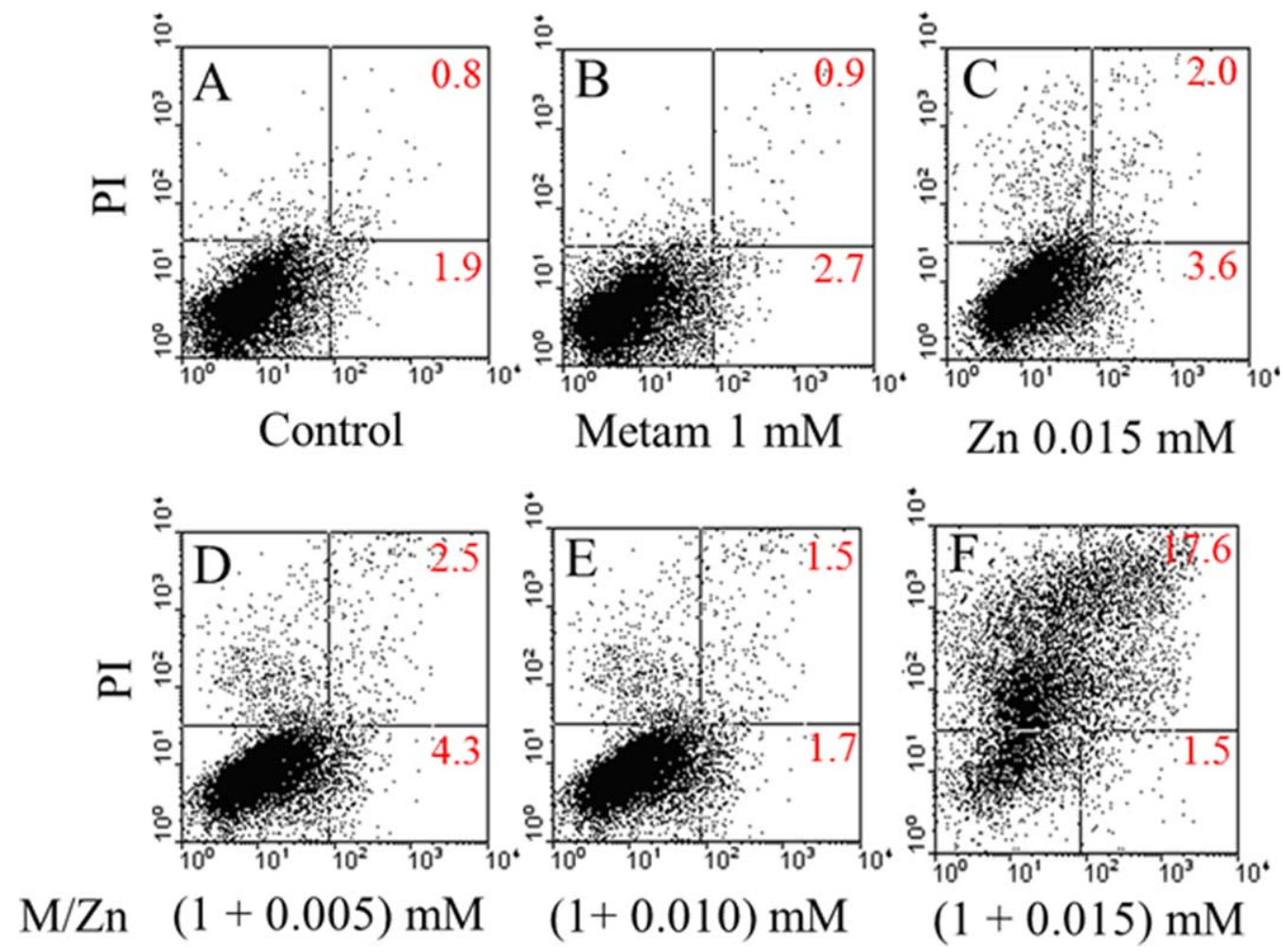

$$
(1+0.015) \mathrm{mM}
$$

Fig. 5. Low-dose effects on necrosis in HepG2 cells treated with $1 \mathrm{mM}$ metam and $(0.005 \sim 0.015) \mathrm{mM}$ zinc (II) for $32 \mathrm{~h}$ by flow cytometry. Each data for three tubes combined. M means metam; M/Cu means the mixture of metam/copper (II); and $\mathrm{M} / \mathrm{Zn}$ means the mixture of metam/zinc (II). [Color figure can be viewed at wileyonlinelibrary.com] 


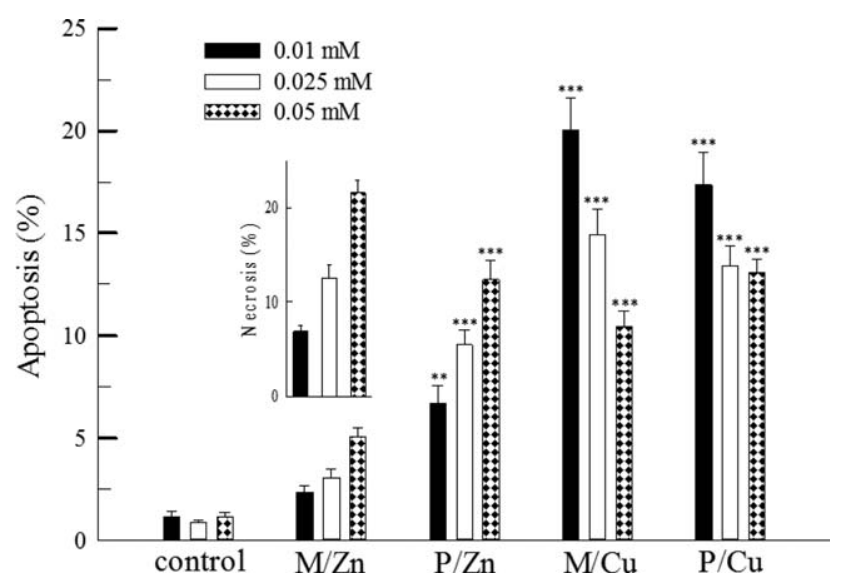

Fig. 6. Early apoptotic/early necrotic distribution (lower-right quadrant area or upper-right quadrant area) in HepG2 cells treated with $0.1 \mathrm{mM}$ metam/PDTC and $(0.01 \sim 0.05) \mathrm{mM}$ copper (II)/zinc (II) for $5 \mathrm{~h}$ by flow cytometry. Each data for three tubes combined. The statistical analysis was followed Turkey-Kramer comparisons at significance level of ${ }^{\star} P<0.05,{ }^{\star \star} P<0.01$, or ${ }^{\star \star \star} P<0.001$ with respect to the corresponding metam/zinc (II) treated cells. $M$ means metam; $P$ means PDTC; $\mathrm{M} / \mathrm{Cu}$ means the mixture of metam/copper (II); M/Zn means the mixture of metam/Zinc (II); P/Cu means the mixture of PDTC/copper (II); and P/Zn means the mixture of PDTC/zinc (II).

apoptotic/early necrotic population (upper-right quadrant, PI-positive/annexin V-positive cells), even more obvious than that apoptotic population in PDTC/zinc (II)-treated HepG2 cells [as shown in Fig. 3(F,I)]. Due to non-toxicity of $(1 \pm 0.05) \mathrm{mM}$ PDTC/copper (II) in HepG2 cells, there was no obvious cell distribution in the area of apoptotic/necrotic quadrants like the control group. Nonetheless, metam/zinc (II)-treated cells in the early apoptotic region (lower-right quadrant, PI-negative/annexinV-positive cells) nearly did not undergo any apoptotic process before they moved into the late apoptotic/early necrotic area [as shown in Fig. 3(G)].

A lag time occurred in apoptotic cells between PS exposure and PI permeability, while in necrotic cells, both events coincide. Therefore, dynamics of apoptosis between PS exposure and PI permeability were distinguished from that of necrosis. Metam/copper (II)-treated cells lay in the early apoptotic region of PI-negative/annexin V-positive at 30 min, subsequently moving toward the late apoptotic region at $60 \mathrm{~min}$, showing an evident time-course change of the typical apoptotic cells [as shown in Fig. 4(E-G)]. Despite the fact that 7.3 percent of the population of metam/zinc (II)-treated cells was in the quadrant of early apoptotic area at $30 \mathrm{~min}$, we also found that considerable numbers of them have entered the region of both early necrotic and late necrotic periods [as shown in Fig. 4(H-J)], possibly undergoing necrotic processes of PS outburst synchronized with membrane permeabilization. Necrosis has long been described as a consequence of extreme high concentration stimuli. Because of the tense physico-chemical stress on the cell, it therefore occurred as an accidental and uncontrolled event. Hence, we attempted to verify whether metam/zinc (II)-treated cells under a low dose could still induce generation of necrotic phenomena. However, as shown in Figure 5, no gradual phase of dose-dependent change from the concentration of $(1+0.01) \mathrm{mM}$ to the concentration of $(1+0.015) \mathrm{mM}$ was captured as assumed in the hypothesis of apoptotic change for low concentration over a relatively long time, but there was a sudden explosively necrotic behavior in HepG2 cells when coincubated with $15 \mu \mathrm{M}$ zinc and $1 \mathrm{mM}$ metam at the 32nd AQ11 hour [Fig. 5(F)]. Then can necrosis indeed occur in the other typical metalchelating dithiocarbamate compounds, such as PDTC/copper (II), PDTC/zinc (II), and metam/copper (II) even though those stimuli were given different concentrations? Both PDTC/copper (II)/or/zinc (II) and metam/copper (II) did not demonstrate such an obvious necrotic phenomenon as metam/zinc cells (as shown in Fig. 6). Preliminarily, metam/ zinc (II)-treated cells were considered undergoing necrotic processes rather than the apoptotic history of metam/copper (II)-treated cells dyed with annexin V-FITC/PI by flow fluorocytometry.

Generally, proliferation and differentiation in normal cells were non-synchronized, where a typical DNA histogram showed a clear diploid DNA peak (2n, G1) during the non-dividing stage and a straight-rising tetraploid peak (4n, G2) during the dividing stage. For apoptotic cells, the formation of apoptotic bodies caused the fragmentation and loss of the DNA, which was therefore characterized by a hypoploid DNA fluorescence pattern of subG0/G1 peaks; necrotic cells maintained their entire DNA content in the nucleus as did the control cells. Thus, the most discriminable feature is the DNA conformation between apoptosis and necrosis in cells. Unexpectedly, in our experimental result, hypoploid DNA distribution of subG0/G1 peaks in metam/zinc (II)-treated cells was similar to the control, however, the DNA content distribution of subG0/G1 peaks in metam/copper (II)-treated HepG2 cells was twofold more than the control (as shown in Fig. 7). In summary, those data gathered by flow fluorocytometry were consistent with morphological changes observed microscopically.

\section{Different Protein Expression Response in HepG2 Cells Between Metam/Copper (II) and Metam/Zinc (II) by Western Blotting}

To further study whether there is a signal transduction pathway apoptosis/necrosis in HepG2 cells between metam/ copper (II) and metam/zinc (II), molecular biological techniques such as real-time fluorescence quantitative PCR (RTPCR) and western blotting were used in our experimental design. The caspase family, the interleukin- $1 \beta$-converting enzyme family proteases, is highly homologous to Caenorhabditis elegans cell death gene CED-3. So far, 14 caspases 


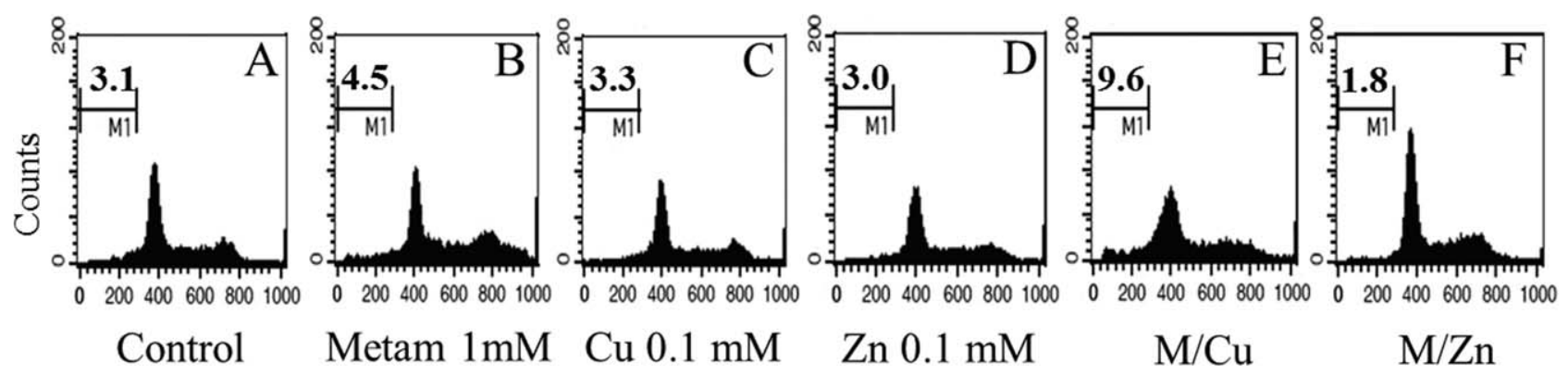

Fig. 7. DNA fragmentation of apoptosis/necrosis-necroptosis at SubGo/G1 peak in HepG2 cells treated with $1 \mathrm{mM}$ metam and $0.1 \mathrm{mM}$ copper (II)/zinc (II) for $1 \mathrm{~h}$ by flow cytometry. Each data for three tubes combined. M means metam; M/Cu means the mixture of metam/copper (II); and M/Zn means the mixture of metam/zinc (II).

have been identified and their precursors are all zymogens known as pro caspases. Caspase 3 can activate DNA fragmentation factor (DFF) when DFFA $(\sim 45 \mathrm{kDa})$ is cleaved and loses its inhibiting function of DNA fragmentation in the apoptotic process. As its chaperone, DFFB $(\sim 40 \mathrm{kDa})$ was found to produce 200bp DNA fragmentation and trigger chromatin condensation during apoptosis. The Bcl-2 family, which also acts on crucial proteins in the regulation of apoptosis, exists in the form of either pro- or anti-apoptotic activities, such as Bax and BCL-2, governing mitochondria outer membrane permeabilization and activating the caspases. Our RT-PCR results showed that those classical apoptotic proteins could be up-regulated in the treated cells compared to untreated control cells (as shown in Fig. S2). Especially in the metam/copper (II)-treated cells, caspase 3, and $\mathrm{Bax} / \mathrm{Bcl}-2$ protein in metam/copper (II)-treated cells were strikingly increased as well as immunoblotting assay auto-activated/activated spontaneously in a chain-like reaction shown in Figure 8, which possibly increased Bax/Bcl-2 upregulated caspase 3 activation (Salakou et al., 2007). In addition, auto-activation of pro caspase-9 also promoted cytochrome $c$ release via binding an apoptotic protease activating factor called apaf1 apoptosome in metam/copper (II)treated cells.

The data, taken together, indicate that the cell death mode induced by metam/copper (II) was through a typical apoptotic pathway, while that induced by metam/zinc (II) was through a typical necrotic/necroptotic pathway.

\section{DISCUSSION}

As the main organ for detoxification in the human body, the liver is responsible for absorption, distribution, metabolism, and elimination of metal-containing complexes. HepG2 lines are well-characterized polarized hepatocyte cells, used in our study as a classic liver cell model. Surprisingly, nontoxic metam could dramatically produce strong synergistic toxicity when combined with copper (II)/zinc (II) in HepG2 cells. Moreover, similar synergistic toxicities in HepG2 cells were observed between the other dithiocarbamates of PDTC, DMDC, and DSF and the metals cadmium (II), chromium (III), nickel (II), manganese (II), and vanadium (II). However, the synergistic toxicities between those dithiocarbamates and copper (II)/zinc (II) were the most remarkable among them. In particular, both dithiocarbamates and copper/zinc-containing compounds are frequently widely applied as medicines and pesticides, thus they probably coexistent in the human body. Therefore, their synergistic toxicities can be occurring in the ecosystem quite broadly. Presently, dose-exposure assessments of pesticides are only performed on a single substance, but our results have indicated that new regulation should be considered and the threshold limits should be further evaluated.

Mammalian cell death has been typically classified as either apoptosis or necrosis. Conceptually, apoptosis is described as an active, regulated, and programmed process of autonomous cellular dismantling; Necrosis is viewed as

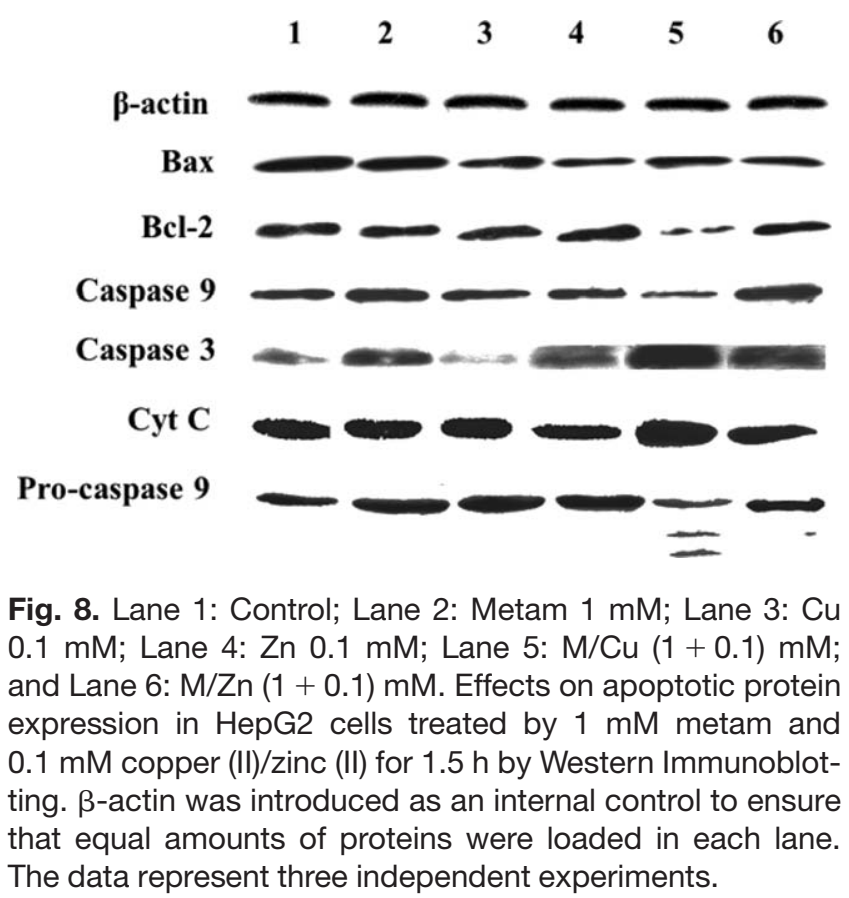


a passive, accidental, and uncontrolled cell death process eliciting inflammation. Principle apoptotic morphology in metam/copper (II) treated cells was characterized as cell cytoplasm shrinkage, membrane blebbing, chromatin condensation, and nuclear fragmentation. By comparison, the main necrotic feature in metam/zinc (II) treated cells was characterized as cell cytoplasm rapid swelling, cell membrane plasma rupture, and organelle breakdown. The other cellular morphologies by flow cytometry (FCM) analysis to distinguish apoptosis and necrosis/necroptosis were early loss of plasma membrane integrity in necrosis/necroptosis of metam/zinc (II) treated HepG2 cells, but its persistence until the late phase in apoptosis of metam/copper (II) treated HepG2 cells. Since there is a lack of specific examples distinguishing either cell apoptosis or necrosis/necroptosis, these verifications probably provide referred regulations a paradigm shift for future study. To test whether necrosis happened in HepG2 cells of metam/zinc (II), identification of biochemical markers and morphological characteristics as well as diverse techniques such as microscopy, flow fluorocytometry, and western blot assay were applied to distinguish apoptosis from necrosis in this paper. Despite the widespread use of the apoptosis/necrosis paradigm, there were no experimental papers that discriminate apoptosis from necrosis through multiple prevailing technologies. Therefore, this is the first reported article that verified two specific examples of apoptosis versus necrosis with versatile combined methods.

In recent decades, light has been shed on differentiating necrosis from apoptosis, which are inherently associated with their respective pathologies. Due to its unwarranted cell loss, necrosis occurs prominently in ischemia, trauma, and neurodegenerative disease, as well as inflammation tumorigenesis. Particularly, necrosis currently has been considered to be finely executed by specialized and programmed signal pathways, and is further defined to be induced by either ligation of the tumor necrosis factor or by caspase inhibition (Galluzzi and Kroemer, 2008), as also shown in our experimental results. Interestingly, one novel theory has pointed that necrotic death and chronic inflammation may foster the onset and growth of tumor (Lotze and DR, 2004). In other words, cancer is one disease that would probably die of necrosis but not apoptosis. Although tumor necrosis factor was originally described as a circulating factor that can induce hemorrhagic necrosis of tumors, it has been found to not only cause the death of cancer cells, but also activate cancer cell survival and proliferation pathways and even promote angiogenesis and tumor cell migration and invasion (Waters et al., 2013). Those effects have shown that necrosis can open one way in cancer therapy by targeting the pathway of tumor necrosis factor.

In addition, as a novel anticancer drug, gold (III) dithiocarbamate compound can inhibit the ubiquitin-proteasome pathway (Milacic et al., 2006). Our initial study showed that both metam/copper (II) and metam/zinc (II) also inhibited the 20S ubiquitin-proteasome pathway. This result will aid in providing new avenues of innovating novel therapeutic medicines for the tumor clinical trials. Presently, attention focused on the cell mode induced by zinc has been gradually raised. Initially, the neuronal death induced by zinc was found to exhibit a feature, more typical of necrosis, which was probably mediated by free radical injury (Kim et al., 1999). The cell death also induced by zinc mixed both necrosis and apoptosis, without caspase-3 activation (Hamatake et al., 2000). Recently, zinc-binding compounds were studied via a distinct mode of action indicating potential cell death followed with necrosis behavior (Ding et al., 2008). Interestingly, It was been found that zinc deficiency can induce apoptosis, while elevated zinc can lead predominately to cell necrosis by generating reactive oxygen species (Kavitha Sankavaram et al., 2010). It is therefore necessary to study that metam/zinc (II) with a specialized necrosis pathway will be worthy investigating its application in the pharmaceutical field.

\section{CONCLUSION}

These results showed that not only metam and copper (II), but also metam and zinc (II) could have significant synergistic toxicities although either of them individually did not appear toxic in HepG2 cells. Further, metam combined with the metals chromium (III), nickel (II), manganese (II), and vanadium (II), also could induce synergistic toxicity in HepG2 cells. Unexpectedly, the death modes of the synergistic toxicities of metam and copper (II)/zinc (II) in HepG2 cells were different: apoptosis induced by metam/copper (II) versus necrosis induced by metam/zinc (II). Moreover, this death mode of metam/zinc (II) in HepG2 cells was also unique compared to the synergistic toxicity between PDTC and zinc (II). Therefore, in this paper, with our understanding based on our studies, necrosis is described for the morphological characteristics induced by metam/zinc in HepG2 cells.

We acknowledged all the members in Professor Benzhan Zhu's group, especially, Chunhua Huang and Qin Hao.

\section{REFERENCES}

US Environmental Protection Agency (USEPA). 2006-2007 Pesticide Market Estimates. U.S. Environmental Protection Agency. http://www.epa.gov/pesticides/pestsales/07pestsales/ table_of_contents2007.htm

Daniel K, Chen D, Orlu S, Cui Q, Miller F, Dou QP. 2005. Clioquinol and pyrrolidine dithiocarbamate complex with copper to form proteasome inhibitors and apoptosis inducers in human breast cancer cells. Breast Canc Res 7:R897-R908.

Ding WQ, Yu HJ, Lind SE. 2008. Zinc-binding compounds induce cancer cell death via distinct modes of action. Canc Lett 271:251-259. 
Duan WR, Garner DS, Williams SD, Funckes-Shippy CL, Spath IS, Blomme EAG. 2003. Comparison of immunohistochemistry for activated caspase- 3 and cleaved cytokeratin 18 with the TUNEL method for quantification of apoptosis in histological sections of PC-3 subcutaneous xenografts. J Pathol 199:221228.

California Environmental Protection Agency. Evaluation of methyl isothiocyanate (MITC) as a toxic air contaminant Part B exposure assessment. Worker health and safety branch department of pesticide regulation. California environmental protection agency, Sacramento, California. http://www.cdpr.ca.gov/ docs/emon/pubs/mitc/augfinl02/augpartb.pdf

Galluzzi L, Kroemer G. 2008. Necroptosis: A specialized pathway of programmed necrosis. Cell 135:1161-1163.

Guicciardi ME, Malhi H, Mott JL, Gores GJ. (2013) Apoptosis and necrosis in the liver. In: Comprehensive Physiology. John Wiley \& Sons. Compr Physiol. 2013 April; 3:(2): doi: 10.1002/ cphy.c120020, 62 pp.

Haendel MA, Tilton F, Bailey GS, Tanguay RL. 2004. Developmental toxicity of the dithiocarbamate pesticide sodium metam in zebrafish. Toxicol Sci 81:390-400.

Hamatake M, Iguchi K, Hirano K, Ishida R. 2000. Zinc induces mixed types of cell death, necrosis, and apoptosis, in molt-4 cells. J Biochem 128:933-939.

Hansen MB, Nielsen SE, Berg K. 1989. Re-examination and further development of a precise and rapid dye method for measuring cell growth/cell kill. J Immunol Meth 119:203-210.

Huang YC, Fan R, Grusak MA, Sherrier JD, Huang CP. 2014. Effects of nano-ZnO on the agronomically relevant Rhizobium-legume symbiosis. Sci Total Environ 497-498:78-90. doi: 10.1016/j.scitotenv.2014.07.100. Epub 2014 Aug 12.

Kavitha S, Leelyn C, Richard SB, Hedley CF. 2010. Zinc deficiency induces apoptosis but zinc induces necrosis in rat hepatoma cells. Faseb J. April 201024 (Meeting Abstract Supplement) 718.3.

Keil DE, Barnes PE, Pruett. DB. SB, 1996. Role of decomposition products in sodium methyldithiocarbamate-induced immunotoxicity. J Toxicol Environ Health 47:479-492.

Kim CH, Kim JH, Xu J, Hsu CY, Ahn YS. 1999. Pyrrolidine dithiocarbamate induces bovine cerebral endothelial cell death by increasing the intracellular zinc level. J Neurochem 72 : 1586-1592.

Kim YH, Kim EY, Gwag BJ, Sohn S, Koh JY. 1999. Zincinduced cortical neuronal death with features of apoptosis and necrosis: Mediation by free radicals. Neuroscience 89:175-182.

Kroupová J, Bártová E, Fojt L, Strašák L, Kozubek S, Vetterl V. 2007. Low-frequency magnetic field effect on cytoskeleton and chromatin. Bioelectrochemistry 70:96-100.

Krysko D, D'Herde K, Vandenabeele P. 2006. Clearance of apoptotic and necrotic cells and its immunological consequences. Apoptosis 11:1709-1726.

Lee S, Harnly RM, Gunier M, Kreutzer RR. 2002. Community exposures to airborne agricultural pesticides in California: Ranking of inhalation risks. Environ Health Perspect 110: 1175-1184.
Linkermann A, Green DR. 2014. Necroptosis. New Engl J Med 370:455-465.

Liu S, Goldstein RH, Scepansky EM, Rosenblatt M. 2009. Inhibition of rho-associated kinase signaling prevents breast cancer metastasis to human bone. Canc Res 69:8742-8751.

Lotze MT,DR. 2004. Dying dangerously: Necrotic cell death and chronic inflammation promote tumor growth. Discov Med 231: 24-33.

Milacic V, Chen D, Giovagnini L, Diez A, Fregona D, Dou QP. 2008. Pyrrolidine dithiocarbamate-zinc (II) and -copper (II) complexes induce apoptosis in tumor cells by inhibiting the proteasomal activity. Toxicol Appl Pharmacol 231:24-33.

Milacic V, Chen D, Ronconi L, Landis-Piwowar KR, Fregona D, Dou QP. 2006. A novel anticancer gold (III) dithiocarbamate compound inhibits the activity of a purified $20 \mathrm{~S}$ proteasome and $26 \mathrm{~S}$ proteasome in human breast cancer cell cultures and xenografts. Canc Res 66:10478-10486.

Nikoletopoulou V, Markaki M, Palikaras K, Tavernarakis N. 2013. Crosstalk between apoptosis, necrosis and autophagy. Biochim Biophys Acta Mol Cell Res 1833:3448-3459.

Nobel CSI, Kimland M, Lind B, Orrenius S, Slater AFG. 1995. Dithiocarbamates induce apoptosis in thymocytes by raising the intracellular level of redox-active copper. J Biol Chem 270: 26202-26208.

Padgett EL, Pruett BD. SB. 1992. Disparate effects of representative dithiocarbamates on selected immunological parameters in vivo and cell survival in vitro in Female B6C3F1 mice. J Toxicol Environ Health 37:559-571.

Pasparakis M, Vandenabeele P. 2015. Necroptosis and its role in inflammation. Nature 517:311-320.

Pruett SB, Han BD, Munson YC. AE. 1992. Immunotoxicological characteristics of sodium methyldithiocarbamates. Fundam Appl Toxicol 18:40-47.

Reynolds P, Gunier VJ, Goldberg PB, Harnly DE, Hertz MA. 2005. Agricultural pesticide use and childhood cancer in California. Epidemiology 16:93-100.

Saeed WK, Jun DW. 2014. Necroptosis: An emerging type of cell death in liver diseases. World J Gastroenterol 20:12526-12532.

Salakou S, Kardamakis D, Tsamandas AC, Zolota V, Apostolakis E, Tzelepi V, Papathanasopoulos P, Bonikos DS, Papapetropoulos T, Petsas T, Dougenis D. 2007. Increased $\mathrm{Bax} / \mathrm{Bcl}-2$ ratio up-regulates caspase-3 and increases apoptosis in the thymus of patients with myasthenia gravis. In Vivo 21: $123-132$.

Tilton F, La Du JK, Vue M, Alzarban N, Tanguay RL. 2006. Dithiocarbamates have a common toxic effect on zebrafish body axis formation. Toxicol Appl Pharmacol 216:55-68.

Tilton F, Tanguay RL. 2008. Exposure to sodium metam during zebrafish somitogenesis results in early transcriptional indicators of the ensuing neuronal and muscular dysfunction. Toxicol Sci 106:103-112.

Waters JP, Pober JS, Bradley JR. 2013. Tumour necrosis factor and cancer. J Pathol 230:241-248. 